\title{
Análise do equilíbrio dinâmico de idosas institucionalizadas após hidrocinesioterapia
}

\author{
Analyze of balance before hydrotherapy intervention
}

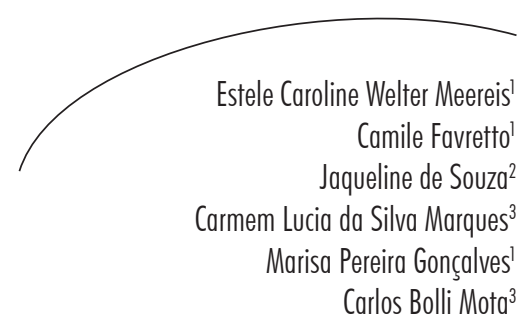

Resumo

Objetivo: O presente estudo teve como objetivo avaliar o equilíbrio dinâmico de idosas institucionalizadas pré e pós-intervenção com hidrocinesioterapia. Método: Para avaliação do equilíbrio foram utilizados dois testes: Escala de Equilíbrio de Berg (EEB) e Performance Oriented Mobility Assessment (POMA). As atividades aquáticas foram realizadas em piscina rasa, uma vez por semana, num total de 15 sessões. Foi aplicado o teste $t$ Student para comparar as avaliações pré e pós-intervenção. Resultados: Observouse melhora na manutenção do equilíbrio corporal no período após a hidrocinesioterapia de forma estatisticamente significativa nas avaliações dos dois testes propostos (EEB p $<0,01$ e POMA p < 0,05). Conclusões: O programa de hidrocinesioterapia demonstrou ser efetivo para a melhora do equilíbrio dinâmico e diminuição do risco de quedas de idosas institucionalizadas. Sendo assim, é um recurso fisioterapêutico que pode ser recomendado para prevenir quedas desta população.

\section{Abstract}

Objective: This study aimed to evaluate the dynamic balance in old women before and after hydrotherapy intervention. Method: To assess the balance, two tests were used: the Berg Balance Scale (BBS) and Performance Oriented Mobility Assessment (POMA). The water activities were conducted in shallow water once a week, totaling 15 sessions. The $t$ Student test was applied to compare the previous and after evaluations. Results: A better body balance after the hydrotherapy intervention was observed being statistically significant in both proposed tests (BBS $\mathrm{p}<0.01$ e POMA $\mathrm{p}<0.05)$. Conclusions: The hydrotherapy program showed to be effective to the dynamic balance and reducing the risk of falls in older women. Therefore, it is a physiotherapy resource which can be recommended to prevent falls of these people.

\footnotetext{
Departamento de Fisioterapia e Reabilitação. Universidade Federal de Santa Maria. Santa Maria, RS, Brasil.

2 Departamento de Fisioterapia. Universidade Federal do Pampa. Uruguaiana, RS, Brasil.

3 Departamento de Esportes Coletivos. Universidade Federal de Santa Maria. Santa Maria, RS, Brasil.

Palavras-chave: Idosos. Envelhecimento. Equilíbrio Postural. Atividade Motora. Marcha.

Key words: Elderly. Aging. Postural Balance. Motor Activity. Gait. 


\section{INTRODUÇÃO}

O equilíbrio corporal requer a capacidade de o indivíduo manter seu centro de gravidade sobre a base de sustentação em situações estática e dinâmicas. Com o envelhecimento, acontece a degeneração gradativa dos sistemas responsáveis pelo equilíbrio, principalmente o visual, vestibular e somato-sensorial.

De acordo com Greve, ${ }^{2}$ a prevalência de queixas de equilíbrio na população idosa chega a $85 \%$, estando associada a várias etiologias, tais como degeneração do sistema vestibular, diminuição da acuidade visual, da capacidade de acomodar a visão e da perseguição uniforme, alterações proprioceptivas, déficits no sistema musculoesquelético, hipotensão postural, atrofia cerebelar, diminuição do mecanismo de atenção e tempo de reação. Esses fatores contribuem para alterações do equilíbrio em indivíduos idosos, associados à diminuição na habilidade em executar as atividades da vida diária (AVDs).

Nesse contexto, surge outro fator de grande relevância epidemiológica, social e econômica, que são as quedas e suas consequências. $\mathrm{Almeida}^{3}$ afirma que a queda é o tipo de acidente mais frequente entre idosos, sendo a principal causa de morte naqueles com mais de 65 anos. Em relação a isso, são conhecidas algumas respostas fisiológicas da atividade em meio líquido, como a melhora na amplitude de movimento, na flexibilidade e na força muscular, também com repercussões psicológicas na promoção de relaxamento, eliminação das tensões mentais, socialização e melhora da autoestima. ${ }^{4}$

As propriedades físicas da água, quando utilizadas como forma de terapia ou coadjuvante na melhora da qualidade de vida de idosos, podem auxiliar na movimentação das articulações, favorecendo a amplitude de movimento e a dinâmica dos sistemas cardiovascular, respiratório e nervoso. ${ }^{5}$
Alguns estudos vêm investigando os efeitos da hidroterapia no equilíbrio e manutenção da postura em idosos. ${ }^{6,7}$ Esses estudos apontam para uma possível melhora na recuperação do equilíbrio, diante de situações instáveis. No entanto, ainda existem especulações em relação à influência da atividade aquática recreativa e terapêutica na melhora do controle postural e, consequentemente, na redução do risco de quedas.

Em vista disso, o presente estudo teve como objetivo avaliar, por meio dos testes EEB e POMA, o equilíbrio dinâmico de idosas pré e pós-intervenção de hidrocinesioterapia.

\section{MÉTODO}

A pesquisa realizada foi aprovada pelo Comitê de Ética e Pesquisa da Universidade Federal de Santa Maria (CAAE-010.0.243.00008/2008), conforme as normas estabelecidas na Resolução no 196/96, do Conselho Nacional de Saúde, sobre pesquisa envolvendo seres humanos, e as participantes assinaram o Termo de Consentimento Livre e Esclarecido.

Foi realizada uma reunião com a diretoria do asilo "Lar das Vovozinhas" do município de Santa Maria para esclarecer o objetivo do estudo, as atividades a serem realizadas e formalizar o convite para sua execução. Inicialmente, 21 idosas concordaram em participar de forma voluntária, e a partir disso foi realizada uma anamnese com as interessadas com o propósito de verificar o enquadramento nos critérios de inclusão e exclusão. Após essa anamnese foi verificado que dez idosas poderiam participar do estudo.

Os critérios de inclusão foram determinados pela idade, a qual deveria ser superior a 60 anos, e disponibilidade de tempo. Os critérios de exclusão foram determinados segundo as principais contraindicações para a prática de atividades aquáticas, tais como febre, erupção cutânea contagiosa, doença infecciosa, doença 
cardiovascular grave, história de convulsões não controladas, hipotensão ou hipertensão grave e incontinência urinária. ${ }^{8}$ Também foi fator de exclusão a incapacidade para manutenção da postura em pé e marcha, a qual se torna necessária para a realização dos testes, além da avaliação realizada por profissional habilitado na área médica (exame médico), oferecido gratuitamente nas dependências do complexo de piscinas do Centro de Educação Física e Desportos da Universidade Federal de Santa Maria, antes do ingresso das idosas e mensalmente durante o período de realização do estudo.

O equilíbrio foi avaliado com base em dois testes: Performance Oriented Mobility Assessment (POMA) e Escala de Equilíbrio de Berg (EEB). A POMA teve o objetivo de detectar fatores de risco de quedas em indivíduos idosos, sendo dividida em duas partes: uma avalia o equilíbrio e outra, a marcha. ${ }^{9} \mathrm{O}$ resultado do teste é obtido por meio do somatório da pontuação alcançada em cada tarefa. O somatório máximo é de 57 pontos, sendo que a pontuação entre 57-41 é considerada equilíbrio normal; entre 37-40, adaptativo; e entre 0-36, anormal, ou seja, severa dificuldade para manutenção do equilíbrio dinâmico.

A fim de se obter mais informações em relação ao equilíbrio das idosas, além da POMA também foi aplicado o teste EEB, que também é embasado na pontuação total obtida.

A EEB faz a descrição quantitativa da habilidade de equilíbrio funcional, ${ }^{9}$ na qual o índice máximo é de 56 pontos, e indica mínimo de risco de quedas, e um índice igual ou menor a 36 está associado a 100\% de risco de quedas. Entre as variações de 56 e 36 pontos, a chance de queda é aumentada em $5 \%$ a cada ponto subtraído.?

A intervenção com hidrocinesioterapia foi realizada em grupo, em piscina rasa $(80 \mathrm{~cm}$ de profundidade), uma vez por semana, num total de 15 sessões, cada uma com duração de 50 minutos. O protocolo de hidrocinesioterapia foi adaptado dos modelos de Resende, Rassi \& Viana. $^{7}$ Campion $^{10}$ e Rauchbach, ${ }^{11}$ sendo composto por exercícios físicos para melhorar a coordenação motora, o equilíbrio, a força muscular, a resistência, a respiração, além de adaptação ao meio líquido e atividades lúdicas e de recreação para proporcionar as idosas um momento de lazer, socialização e descontração.

Para a análise estatística, foi utilizado o teste $t$ Student para os valores obtidos na EEB e POMA antes e após a intervenção hidrocinesioterapêutica, para os quais foi adotado $\mathrm{p}<0,05$ e $\mathrm{p}<0,01$

\section{RESULTADOS}

O grupo de estudo foi composto por dez idosas, das quais foram mensuradas as medidas de massa e estatura corporal. Os valores obtidos estão ilustrados na tabela 1 .

Tabela 1 - Análise descritiva dos dados referentes às características do grupo. Santa Maria, RS, 2010.

\begin{tabular}{cl}
\hline $\mathrm{n}=10$ & Média (DP) \\
\hline Idade (anos) & $62,12 \pm 2,94$ \\
Massa $(\mathrm{kg})$ & $75,34 \pm 7,55$ \\
Estatura (m) & $1,54 \pm 0,03$ \\
IMC $\left(\mathrm{kg} / \mathrm{m}^{2}\right)$ & $31,50 \pm 3,63$ \\
\hline
\end{tabular}

IMC: índice de massa corporal; DP: desvio-padrão. 
Em relação à análise do equilíbrio dinâmico, a maioria das idosas apresentou maiores escores, o que infere um menor risco de quedas, na avaliação após a intervenção.
A figura 1 ilustra o somatório obtido por cada idosa no teste POMA de equilíbrio nos períodos pré a pós-intervenção com hidrocinesioterapia.

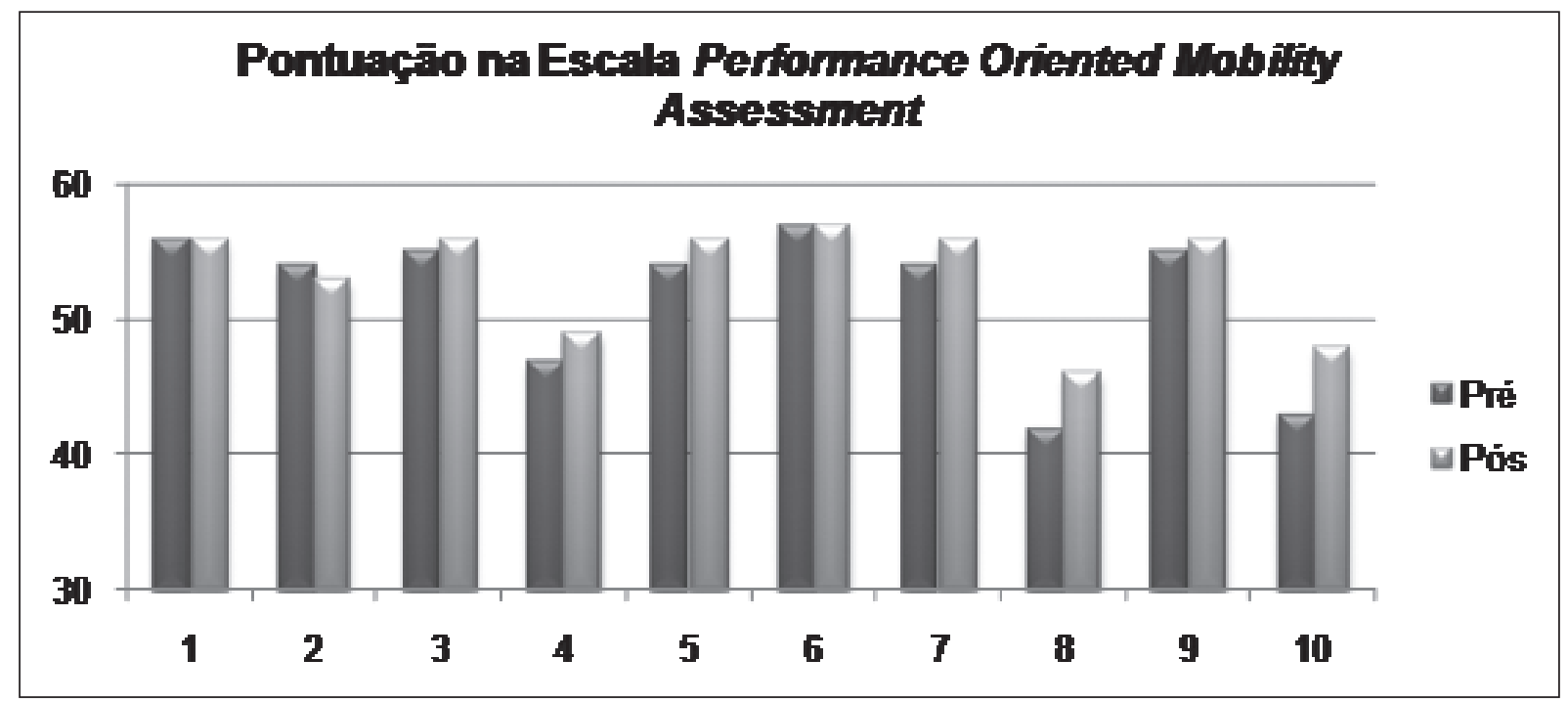

Figura 1 - Pontuação obtida por cada idosa na escala de equilíbrio POMA nas avaliações pré e pós o tratamento proposto. Santa Maria, RS, 2010.

$\mathrm{Na}$ figura 1, é possível observar que, por meio da avaliação da POMA, 60\% das idosas aumentaram a pontuação - ou seja, melhoraram seu equilíbrio corporal na situação pós-tratamento, comparado ao teste realizado pré-tratamento; e
$40 \%$ obtiveram, na avaliação pós, escores menores ou iguais aos da avaliação pré-tratamento.

A figura 2 ilustra o somatório obtido por cada idosa no teste EEB nos períodos pré e pós a intervenção com hidrocinesioterapia.

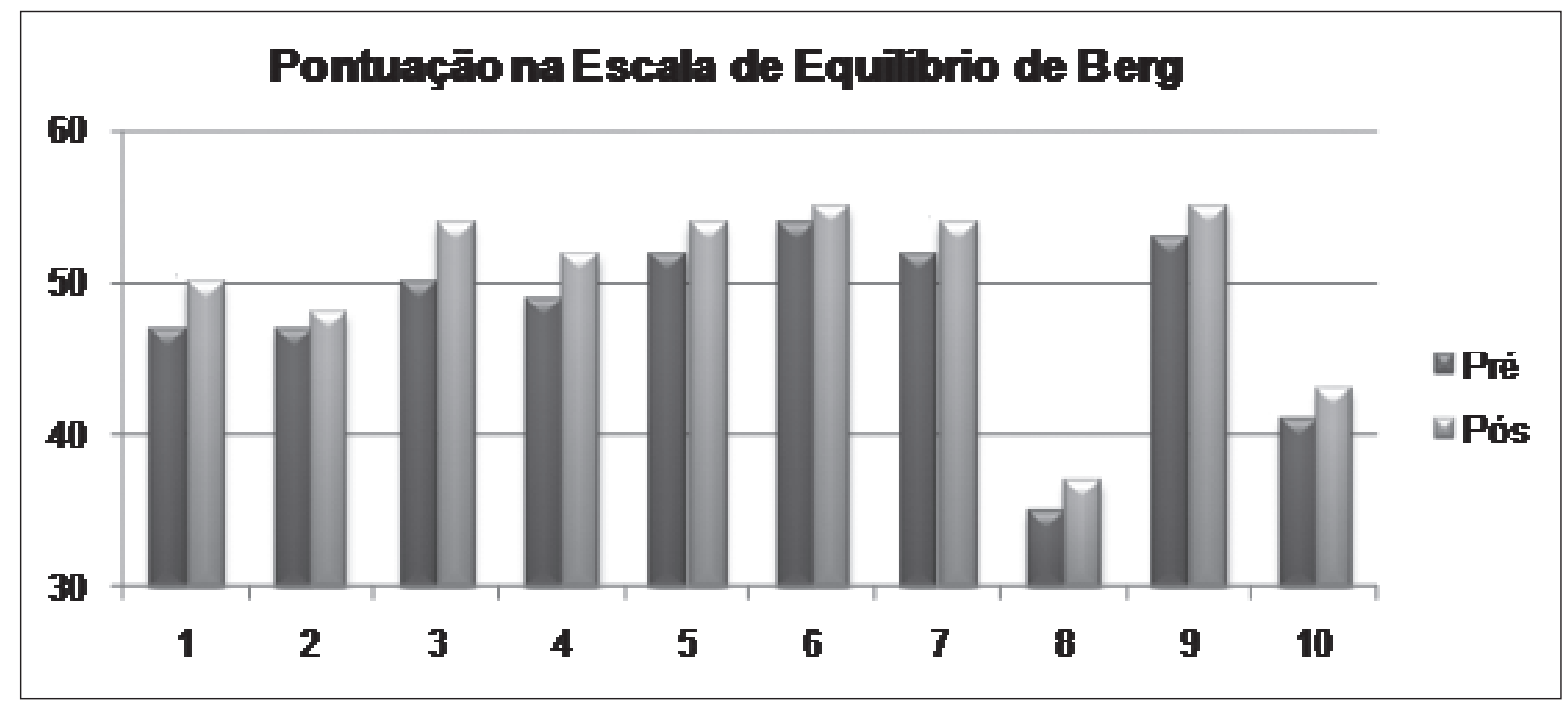

Figura 2 - Pontuação obtida por cada idosa na escala de equilíbrio de Berg na avaliação pré e pós o tratamento proposto. Santa Maria, RS, 2010. 
Como pode ser observado na figura 2 , quando avaliadas utilizando-se a EEB, $100 \%$ dos sujeitos investigados aumentaram o somatório no teste, o que infere uma melhora no equilíbrio dinâmico.
Para verificar se a melhora do equilíbrio apresentada no período pós-tratamento na avaliação com os testes POMA e EEB foi estatisticamente significativa em relação ao período pré, aplicou-se o teste $t$ Student, e os valores obtidos estão ilustrados na tabela 2 .

Tabela 2 - Análise comparativa dos dados referentes ao equilíbrio no período pré e pós-tratamento. Santa Maria, RS, 2010.

\begin{tabular}{lccc}
\hline & $\begin{array}{c}\text { Avaliação Pré } \\
\text { Média (DP) }\end{array}$ & $\begin{array}{c}\text { Avaliação Pós } \\
\text { Média (DP) }\end{array}$ & p-value \\
\hline POMA & $51,7 \pm 5,54$ & $53,3 \pm 4,08$ & $0,02237^{*}$ \\
EEB & $48,0 \pm 5,94$ & $50,2 \pm 5,99$ & $0,00003 \dagger$ \\
\hline
\end{tabular}

POMA: Performance Oriented Mobility Assessment; EEB: Escala de Equilíbrio de Berg; $\mathrm{p}$-value $=$ valor de probabilidade no teste $t$ Student; $*$ Indica diferença estatisticamente significativa, $\mathrm{p}<0,05$; $†$ Indica diferença estatisticamente significativa, $\mathrm{p}<0,01$.

Comparando-se os valores obtidos pelos dois testes, pré e pós a intervenção, nota-se que houve melhora estatisticamente significativa na manutenção do equilíbrio corporal. Em relação à EEB, a diferença foi mais significativa $(\mathrm{p}<$ $0,01)$, se comparada a POMA ( $<<0,05)$.

\section{DISCUSSÃO}

De acordo com os resultados obtidos por meio dos dois testes utilizados no presente estudo, o equilíbrio melhorou de forma estatisticamente significante após a execução do programa de hidrocinesioterapia proposto. O mesmo ocorreu nos estudos conduzidos por outros autores, ${ }^{7,12-15}$ nos quais a aplicação de um programa de hidroterapia diminuiu o risco de quedas de populações idosas. Não há, entretanto, uma padronização dos testes funcionais utilizados para avaliação do equilíbrio de idosos, o que dificulta a comparação quantitativa.

Karuka et al. $^{16}$ analisaram a concordância entre instrumentos de avaliação do equilíbrio corporal de idosos e verificaram que existe correlação dos testes utilizados no presente estudo, teste POMA e EEB, e desses testes com demais instrumentos, como o Teste de Alcance Funcional Anterior (TAF).

Neste estudo, foi possível observar que houve semelhança nos resultados demonstrados pelo POMA e EEB, como por exemplo, o sujeito oito, que apresentou menor escore nos dois testes; assim como os sujeitos seis e nove que apresentaram elevados escores nos dois testes. Pela avaliação do EEB, no entanto, todos os sujeitos melhoraram seus índices de manutenção do equilíbrio, e pelo teste POMA, apenas $60 \%$. Diante disso, verifica-se a importância da aplicação conjunta de diferentes instrumentos de avaliação para melhor avaliar o equilíbrio de idosas, como afirmam Karuka et al. ${ }^{16}$

Ao classificar a pontuação das idosas institucionalizadas de acordo com a EEB, uma idosa apresentou elevado risco de quedas. Já em relação à POMA, todas as idosas tiveram pontuação suficiente para ser classificadas em equilíbrio normal.

Pode ser observado que a atividade proposta aumentou os escores de idosas que apresentavam diferentes níveis de risco de queda, e portanto podem ser indicados para diversas populações de idosos. Os exercícios de hidrocinesioterapia 
são indicados pelo fato de o meio líquido proporcionar maior segurança na realização dos movimentos, prolongando o tempo de retomada da postura quando o corpo se desequilibra. Assim, pode-se desafiar o indivíduo além de seus limites de estabilidade, sem temer as consequências de queda que podem ocorrer no solo. ${ }^{17,18}$

Segundo Geigle et al., ${ }^{17}$ os exercícios físicos são vistos como uma forma de melhorar a condição de equilíbrio, pois favorecem o aprimoramento das condições de recepção de informações sensoriais do sistema vestibular, visual e somato-sensorial, e ainda ativam os músculos antigravitacionais, reestruturando, por sua vez, o equilíbrio.

Douris et al., ${ }^{14}$ em seu estudo, verificaram que tanto o grupo que realizou atividades em piscina quanto em solo obtiveram melhora nos escores referentes à EEB. Por outro lado, Cunha et al. ${ }^{19}$ verificaram que o equilíbrio e mobilidade aumentaram no grupo que realizou fisioterapia aquática, enquanto o grupo que realizou fisioterapia em solo não apresentou melhora de acordo a EEB. Diante disso, pode ser observado que não há consenso entre os autores em relação aos benefícios da atividade realizada em solo para o equilíbrio de idosos, enquanto em ambos os estudos a atividade realizada em meio líquido é referida como eficiente para diminuir o risco de quedas de idosos.

Parece existir consenso entre os autores quanto àindicação de exercícios aquáticos para indivíduos com risco de quedas. ${ }^{7,13-15}$ As propriedades físicas da água, somadas aos exercícios, podem cumprir a maioria dos objetivos físicos propostos num programa de reabilitação. ${ }^{19} \mathrm{O}$ meio liquido é apropriado para a prática de hidroterapia de pessoas idosas, pois permite o atendimento em grupo e facilita a recreação, a socialização e a movimentação de forma mais segura, e estas, associadas a melhoras funcionais, melhoram a autoestima e a autoconfiança do idoso. ${ }^{6}$

Muitas vezes há insegurança, por parte dos idosos, em relação ao meio líquido, em função da profundidade da água, o que os faz preferir atividades em solo. Alguns dos estudos referidos não apresentam o nível de profundidade da água utilizado, como o de Cunha et al. ${ }^{19} \mathrm{O}$ presente estudo, no entanto, demonstra que não é necessário um nível de profundidade elevado, pois a hidrocinesioterapia realizada em piscina rasa foi eficaz para melhorar o equilíbrio do grupo investigado.

Observa-se que a hidroterapia pode trazer diversos benefícios para indivíduos idosos e, diante dos resultados obtidos, foi possível verificar que a hidrocinesioterapia realizada em piscina rasa pode trazer benefícios relacionados ao equilíbrio e diminuir o risco de quedas em idosos institucionalizados. Portanto, pode ser uma ferramenta para tratamento e prevenção de quedas nessa população.

\section{CONCLUSÕES}

O programa de hidrocinesioterapia proposto demonstrou ser efetivo na redução do risco de quedas da maioria das idosas institucionalizadas participantes do estudo. Os resultados referentes a POMA demonstraram que $60 \%$ das idosas melhoraram seu equilíbrio dinâmico; já aqueles referentes a EEB demonstraram que 100\% das idosas melhoraram seu equilíbrio dinâmico.

Diante disso, observa-se a importância de se utilizar mais de um teste durante as avaliações. Apesar de o grupo de estudos ter sido pequeno e de não analisarmos um grupo controle, os resultados indicam que os programas de exercícios de hidroterapia são capazes de aumentar o equilíbrio dinâmico e reduzir o risco de quedas em idosas institucionalizadas. Sendo assim, é um possível recurso fisioterapêutico recomendado para prevenir as quedas dessa população.

\section{AGRADECIMENTOS}

Agradecemos a colaboração das monitoras que acompanharam as atividades de hidrocinesioterapia: Carla Francieli Spohr, Ivone von Mulen, Matiele Leal Bueno e Natália San Martin dos Santos. 


\section{REFERÊNCIAS}

1. Daniel F, Vale R, Giani T, Bacellar S, Dantas E. Effects of a Physical Activity Program on Static Balance and Functional Autonomy in Elderly Women Macedonian. Maced J Med Sci 2010;3(1):21-6.

2. Greve P, Guerra AG, Portela MA, Portes MS, Rebelatto JR. Correlações entre mobilidade e independência funcional em idosos institucionalizados e não-institucionalisados. Fisioter Mov 2007;20(4):117-24.

3. Almeida ST. Análise da estabilidade postural de idosos sedentarismo, praticantes de exercício físico e atletas. RBCEH 2007;4(1):39-47.

4. Marques J, Pereira N. Hidroginástica: exercícios comentados, cinesiologia aplicada à hidroginástica. Rio de Janeiro: Pereira; 1999.

5. Rocha JCC. Hidroginástica Teoria e Prática. 2a ed. Rio de Janeiro: Sprint; 1994.

6. Candeloro JM, Caromano FA. Efeito de um programa de hidroterapia na flexibilidade e na força muscular de idosas. Rev Bras Fisioter 2007;11(4):303-9.

7. Resende SM, Rassi CM, Viana FP. Efeitos da hidroterapia na recuperação do equilíbrio e prevenção de quedas em idosas. Rev Bras Fisioter 2008;12(1):57-63.

8. Biasoli MC, Machado CMC. Hidroterapia: técnicas e aplicabilidades nas disfunções reumatológicas. Temas de Reumatologia Clínica 2006;7(3):78-87.

9. Figueiredo KMOB, Lima KC, Guerra RO. Instrumentos de avaliação do equilíbrio corporal em idosos. Rev Bras Cineantropom Desempenho Hum 2007;9(4):408-13.

10. Campion MR. Hidroterapia: princípios e prática. São Paulo: Manole; 2000.
11. Rauchbach RA. Atividade física para a terceira idade, analisada e adaptada. Curitiba: Lovise; 1990.

12. Shumway-Cook A, Baldwin M, Polissar NL, Gruber W. Predicting the probability for falls in communitydwelling older adults. Phys Ther 1997;77(8):812-9.

13. Booth CE. Water exercise and its effects on balance and gait to reduce the risk of falling in older adults. Act Adapt Aging 2004;8(4):45-57.

14. Douris P, Southard V, Varga C, Schauss W, Gennaro C, Reiss A. The effect of land and aquatic exercise on balance score in older adults. J Geriatr Phys Ther 2003;26(1):3-6.

15. Lord SR, Matters B, George RS, Thomas M, Bindon J, Chan DK, et al. The effects of water exercise on physical functioning on older people. Aust J Ageing. 2006;25(1):36-41.

16. Karuka AH, Silva JAMG, Navega MT. Análise da concordância entre instrumentos de avaliação do equilíbrio corporal em idosos. Rev Bras Fisioter 2011;15(6):460-6.

17. Geigle PR, Cheek WL, Gould ML, Hunt HC, Shaiq B. Aquatic physical therapy for balance: the interaction of somatosensory and hydrodynamic principles. The J Aquatic Phys Ther 1997;5(1):4-10.

18. Salzman AP. Evidence-based aquatic therapy for proprioceptive-training. The Aquatic Resources Network. Atri's Aquatic Symposium 1998:H95-9.

19. Cunha MF, Lazzareschi L, Gantus MC, Suman MR, Silva A, Parizi CC, et al. A influência da fisioterapia na prevenção de quedas em idosos na comunidade: estudo comparativo. Motriz Rev Educ Fis 2009;15(3):527-36. 